\title{
Orientational states and phase transitions induced by microtextured substrates
}

\author{
Tie-Zheng Qian and Ping Sheng \\ Department of Physics, The Hong Kong University of Science and Technology, Clear Water Bay, Kowloon, Hong Kong
}

(Received 27 February 1997)

\begin{abstract}
We study the orientational states induced in nematic liquid crystals by microtextured substrates with a spatially mixed pattern of different aligning potentials on the mesoscopic scale, i.e., on the order of $0.5 \mu \mathrm{m}$ or less. We show that within the Landau-de Gennes framework, the existence of a finite elastic correlation length leads to the prediction of multiple (temperature-dependent) bulk orientational states, separated by phase transition(s). For an alternating stripe pattern of planar and homeotropic aligning potentials, we find three orientational states, denoted by the alignment of their bulk directors as the $x$, the $y$, and the $y z$ states. Here $z$ is normal to the substrate, $y$ is parallel to the stripes, and the texturing is along the $x$ direction. In particular, there is a first order phase transition between the two states with the bulk directors in the $x$ direction and in the $y z$ plane. This transition can be induced by varying either the texturing periodicity or the temperature, or through the application of an electric field in the $z$ direction. For nematic liquid crystals sandwiched between two similarly textured substrates, an analysis of the electric-field-induced effects shows that the effective anchoring strength of the textured substrates is tunable as a function of either the texturing periodicity or the temperature. When the anchoring is weak, a field-induced orientational transition is found to be possible at applied voltages lower than that of the Fréedericksz transition. [S1063-651X(97)10906-0]

PACS number(s): 64.70.Md, 61.30.Cz, 68.45.- -v
\end{abstract}

\section{INTRODUCTION}

The study of substrate-induced alignments is important for both the physical understanding of substrate-liquidcrystal (LC) interactions and the design of LC display devices. Properly treated substrates are known to induce preferential bulk alignment in LCs. Most prior studies on the subject have been focused on uniform substrates. Perfectly uniform substrates are rare, however, and conventionally by uniform substrates one usually means those samples where the microstructure scale is much smaller than the LC elastic correlation length, to be defined below (Sec. III).

For a uniform substrate, substrate-LC interaction can result in liquid crystalline order within the boundary layer, close to the substrates, even when there is no bulk ordering at temperatures above the nematic-isotropic transition temperature $T_{N I}$. Below $T_{N I}$ the effect of a uniform substrate is mostly to anchor the bulk director through a boundary layer that exhibits a slight variation of liquid crystalline order. The LC elastic correlation length serves as the basic length scale for the imperfectly ordered interfacial layer. There have been a large number of theoretical and experimental studies on such local ordering near the substrates. Close to and above $T_{N I}$ it has been shown that there can be three phenomena associated with the boundary layer: (1) partial and complete wetting, (2) the boundary layer phase transition, and (3) the biaxial state. In (1), the partially ordered boundary layer is viewed as the wetting of the interface by an ordered LC phase. As the temperature approaches $T_{N I}$ from above, the thickness of the ordered layer can be either finite (partial wetting) or infinite (complete wetting), depending on the relative strength of the LC-substrate interaction. These two distinct cases have both been experimentally observed [1]. It has also been predicted that a phase transition, of first or second order, can occur in an ordered boundary layer at a temperature above $T_{N I}$ if the LC-substrate interaction is within the appropriate range [2,3]. This phenomenon, also called the prewetting transition, is, to the best our knowledge, yet to be observed. For phenomenon (3), it has been predicted and experimentally observed that biaxial ordering can exist in the boundary layer $[3,4]$. By biaxiality, one means that there can be two nonzero order parameters along two local orthogonal axes. In mathematical terms this means that in the principal local frame where the tensor order parameter $\stackrel{\leftrightarrow}{Q}$ is diagonal, there are two independent diagonal elements. Theoretical studies have shown that above $T_{N I}$, strong planar alignment potential can cause spontaneous symmetry breaking that results in biaxial ordering in the boundary layer [3]. A recent experiment [4] has indeed found that the first LC monolayer at the polyimide-coated substrate can possess strong biaxial character, which must be described by a tensor order parameter. The use of the tensor order parameter in this case proves to be not only necessary, but also sufficient for the accurate prediction of the bulk orientational state through the Landau-de Gennes formalism - the predicted and measured bulk tilt angles were shown to agree accurately [4], demonstrating the validity and accuracy of the phenomenological model based on the tensor order parameter. In this work, the Landau-de Gennes formalism is the basis of all our calculations.

In contrast with prior studies on uniform substrates, this work is concerned with phenomena that can be induced by microtextured substrates, i.e., sample substrates where the microstructure scale is purposely made larger than the LC elastic correlation length, but still in the mesoscopic range. In this context, it should be mentioned that there have been prior works that examined the effective alignment induced by inhomogeneous substrates [5-7]. Berreman [5] first studied the anchoring effect of a periodically undulating surface where the surface anchoring is locally strong. Faetti [6] generalized Berreman's results by taking into account the mag- 
nitude of the local anchoring energy. Barbero et al. [7] further considered a flat solid surface textured into a periodic structure along one direction in the plane, with two alternating easy axes. They showed that an effective easy axis can result for bulk alignment. However, all those prior studies on inhomogeneous substrates were based on the Frank-Oseen elastic theory, which considers only the distortion energy associated with the spatial variation of the director. The neglect of the spatial variation of the liquid crystalline order means that the elastic correlation length is essentially infinite in the Frank-Oseen theory [8]. That means the substrate inhomogeneity is effectively homogenized, and the effective alignment is the only phenomenon that can result.

In this work, we consider, besides the distortion energy of the directors, also that associated with the spatial variation of the liquid crystalline order parameter. It is shown that within the more complete and accurate theoretical framework, there can be more than a single (temperature-dependent) bulk alignment state, together with phase transition(s) between the induced states. Moreover, the effective anchoring strength can be tuned, giving rise to field-induced effects at voltage below the Fréedericksz transition voltage.

\section{STATEMENT OF RESULTS}

We consider the possible orientational states that can be induced by micro-textured substrates with a spatially mixed pattern of different aligning potentials on the mesoscopic scale, i.e., on the order of $0.5 \mu \mathrm{m}$. For a stripe pattern of alternating planar and homeotropic aligning conditions, shown in Fig. 1(a), we find that there are three orientational states [9]. In each of these states, close to the substrate is a boundary layer, whose thickness is proportional to the periodicity of the textured pattern and that exhibits appreciable elastic distortion. Above the boundary layer is the bulk region where the liquid crystalline order is uniaxial with a uniform bulk director. The three states, denoted as the $x$, the $y$, and the $y z$ states, have their bulk directors in the $x$ and the $y$ directions, and in the $y z$ plane, respectively. The director configurations of the $x$ state and the $y z$ state are schematically illustrated in Fig. 1. Here $z$ is normal to the substrate, $y$ is parallel to the stripes, and the stripe texturing is along the $x$ direction. In the $y z$ state, the tilt angle of the bulk director, as measured from the $x y$ plane, can vary between 7 and $40^{\circ}$ as a function of temperature and/or texturing periodicity. The existence of those states is found to result from the competition between the elastic energy in the boundary layer, induced by the microtexturing, and the surface alignment potential. When the texturing periodicity $P$ is very large as compared to the elastic correlation length, the elastic energy is small, and the surface alignment potential dominates the behavior in the boundary layer. This leads to the $y z$ state. When $P$ decreases, the elastic energy can increase to the extent where it is comparable to the surface alignment potential. Further decrease of $P$ results in the unfavorably large elastic energy, and a transition becomes possible to state(s) where the elastic distortion is small, at the expense of disobeying the surface alignment potential. Two states are found to be possible: the $x$ state and the $y$ state. Through an analysis of the rotational and space-reversal symmetry properties of the system, coupled with a simple model, it is
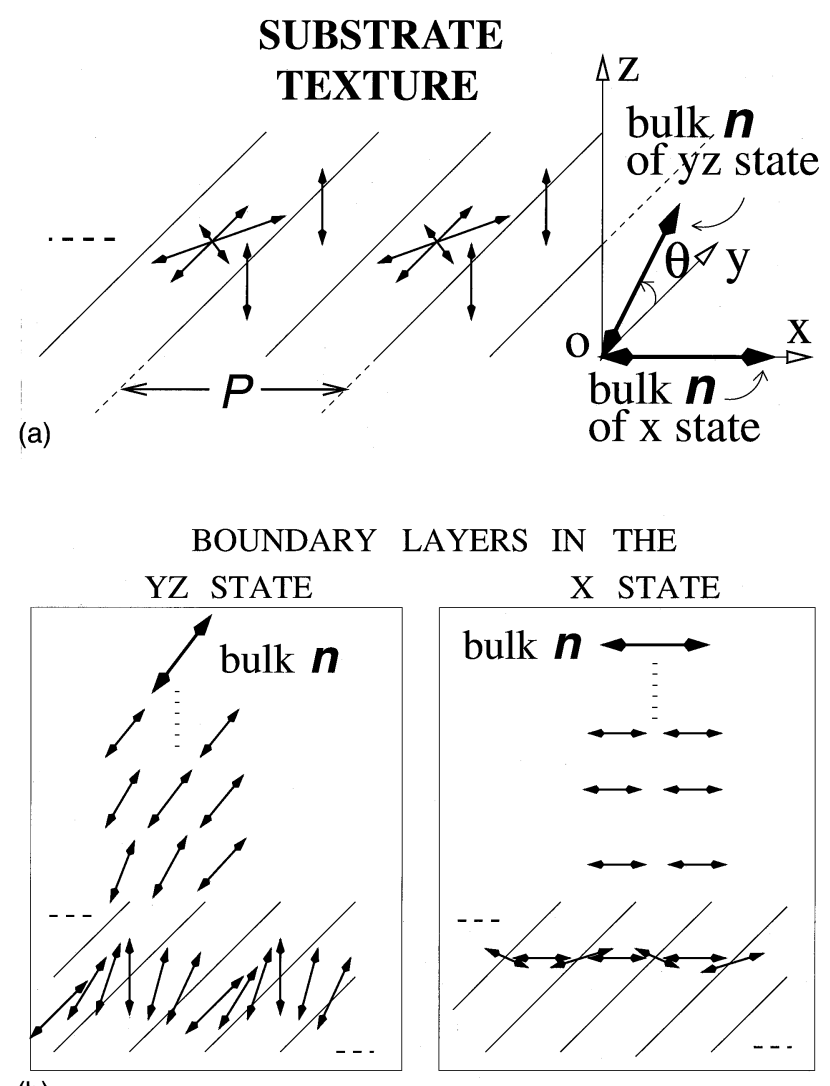

(b)

FIG. 1. Schematic illustration of the textured stripe pattern with alternating homeotropic and planar alignment conditions. The directors of the two bulk orientational states are shown at right. (b) The director configurations of the $y z$ state and the $x$ state in the transition layer.

shown that the $x$ state is the stable phase and the $y$ state the metastable one. Furthermore, we find that there can be a first order phase transition between the $x$ and the $y z$ states. The transition can be observed by varying either the temperature or the texturing periodicity, or through the application of an electric field in the $z$ direction. The field-induced transition in a sandwiched LC cell is found to be possible at a voltage lower than that of the Fréedericksz transition, i.e., when the field-induced distortion length $\xi_{E}$ is larger than the cell thickness $d$. This is shown to be possible because the existence of the induced phases implies that close to the transition point, the surface anchoring is effectively weak.

This paper is organized as follows. In Sec. III we present the Landau-de Gennes formalism for our model. In Sec. IV the numerical procedure for locating the stable and the metastable states is outlined, and the existence of three bulk orientational states, namely, the $x$ state, the $y$ state, and the $y z$ state, is shown. By examining the large and small periodicity limits, we explain why there can be three locally and potentially globally stable states. In Sec. V, we demonstrate the existence of a first order phase transition from the $y z$ state to the $x$ state upon the variation of the substrate periodicity and/or the temperature. For completeness, the virtual transition from the metastable $y z$ state to the metastable $y$ state is also analyzed. In Sec. VI, we discuss the effects of applied electric field on nematic liquid crystals sandwiched between two similarly microtextured substrates. With appro- 
priate surface potential constants, it is shown that when the cell thickness is on the order of several micrometers, an applied voltage on the order of a few volts is sufficient to induce a first order transition from the $y z$ state to the $x$ state, provided the starting state is not far from the transition point. The field-induced phase transition effectively implies that microtexturing offers a systematic approach to continuously vary the anchoring strength. We conclude in Sec. VII with a discussion of future works.

\section{THE MODEL}

Consider a semi-infinite nematic liquid crystal (NLC) sample interfaced with a substrate that is textured into a periodic stripe pattern. The system is in the half space $z \geqslant 0$ and the substrate is defined to be at $z=0$. In the Landau-de Gennes formalism, the local ordering of a NLC is macroscopically described by a second-rank traceless, symmetric tensor order parameter $\overleftrightarrow{Q}$, which has five independent components. Expansion of the free energy density in a power

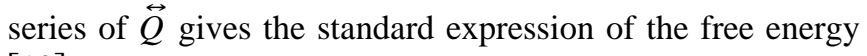
[10]:

$$
\begin{aligned}
F_{B}= & \int d^{3} \mathbf{r} \frac{1}{2}\left(\alpha Q_{i j}^{2}+L_{1} Q_{i j, k}^{2}+L_{2} Q_{i j, j} Q_{i k, k}\right)-\beta Q_{i j} Q_{j k} Q_{k i} \\
& +\gamma\left(Q_{i j}^{2}\right)^{2}
\end{aligned}
$$

Here $\alpha, L_{1}, L_{2}, \beta$, and $\gamma$ are phenomenological constants, the indices $i, j, k$ running from 1 to 3 with the correspondence $1 \leftrightarrow x, 2 \leftrightarrow y, 3 \leftrightarrow z$, summation over repeated $i, j, k$ indices is implied, and the comma in the subscript means derivative with respect to the spatial coordinate that follows. $\stackrel{\leftrightarrow}{Q}$ can be diagonalized at any spatial point $\mathbf{r}$. If two of the three diagonal elements are equal, then the local ordering is uniaxial and $\stackrel{\leftrightarrow}{Q}$ can be written as $S(3 \mathbf{n n}-1) / 2$ where $S$ reflects the uniaxial liquid crystalline order and $\mathbf{n}$ is the director. To relate the phenomenological constants to those conventionally encountered in the uniaxial case, we substitute $\overleftrightarrow{Q}=S(3 \mathbf{n n}-1) / 2$ into Eq. (1) to yield

$$
\begin{aligned}
f_{B}^{U}= & a\left(T-T^{*}\right) S^{2}-B S^{3}+C S^{4}+\frac{3}{4}\left(L_{1}+\frac{1}{6} L_{2}\right)[\nabla S]^{2} \\
& +\frac{3}{8} L_{2}[\mathbf{n} \cdot \nabla S]^{2}+\frac{9}{4} S^{2}\left\{\left(L_{1}+\frac{1}{2} L_{2}\right)[\nabla \cdot \mathbf{n}]^{2}\right. \\
& \left.+L_{1}[\mathbf{n} \cdot \nabla \times \mathbf{n}]^{2}+\left(L_{1}+\frac{1}{2} L_{2}\right)[\mathbf{n} \times \nabla \times \mathbf{n}]^{2}\right\} \\
& +\frac{3}{2} L_{2} S[\nabla \cdot \mathbf{n}][\mathbf{n} \cdot \nabla S]+\frac{3}{4} L_{2} S[\mathbf{n} \times \nabla \times \mathbf{n}] \cdot \nabla S,
\end{aligned}
$$

where $a\left(T-T^{*}\right)=3 \alpha / 4, T^{*}$ is the supercooling temperature, $B=3 \beta / 4$, and $C=9 \gamma / 4$. In case $\mathbf{n}(\mathbf{r})$ is uniform, only the first five terms in Eq. (2) survive. The sixth term is expressed in the familiar form of splay, $[\nabla \cdot \mathbf{n}]^{2}$, twist, $[\mathbf{n} \cdot \nabla \times \mathbf{n}]^{2}$, and bend, $[\mathbf{n} \times \nabla \times \mathbf{n}]^{2}$, distortions, concerning the spatial variation of $\mathbf{n}(\mathbf{r})$, with respective elastic constants given by $K_{\text {splay }}=\frac{9}{2} S^{2}\left(L_{1}+1 / 2 L_{2}\right), \quad K_{\text {twist }}=\frac{9}{2} S^{2} L_{1}, \quad$ and
$K_{\text {bend }}=\frac{9}{2} S^{2}\left(L_{1}+\frac{1}{2} L_{2}\right)$. The last two terms represent the coupling between the spatial variations of $\mathbf{n}(\mathbf{r})$ and $S(\mathbf{r})$. From Eqs. (1) and (2), it is clear that one can define a length scale from the ratio between the coefficient(s) of the gradient terms and those of the nongradient term(s). Such length scale(s) is (are) denoted the elastic correlation length(s) in this paper.

In the absence of any surface interaction potential, the bulk state in equilibrium is uniform. Minimizing $F_{B}$ with respect to a uniform $\overleftrightarrow{Q}$ (r) yields the uniaxial state characterized by an orientational order parameter $S$ and a director $\mathbf{n}$. For the semi-infinite sample in $z \geqslant 0$, the equilibrium bulk state at $z \rightarrow \infty$ must be uniform and thus uniaxial, since otherwise the elastic distortion energy would diverge. However, when a NLC interacts with the substrate at $z=0$ via a surface interaction potential, deviation in the surface order parameter

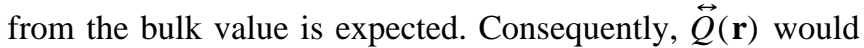
inevitably exhibit spatial variation close to the substrate. Several elastic correlation lengths of the same order can be defined from Eq. (1). For a uniform substrate, the spatial variation of $\stackrel{\leftrightarrow}{Q}(\mathbf{r})$ in the $z$ direction exists only in the boundary layer, with the LC elastic correlation length,

$$
\Delta=\sqrt{\frac{L C}{B^{2}}},
$$

as its characteristic length unit, where $L=\frac{3}{4} L_{1}+\frac{1}{2} L_{2}$.

Assuming the stripe pattern at textured substrate is periodic in the $x$ direction, we write the surface interaction potential as

$$
F_{S}=\int d^{3} \mathbf{r} f_{S}[\stackrel{\leftrightarrow}{Q}(\mathbf{r}), x] \delta(z)
$$

in which $f_{S}[\overleftrightarrow{Q}, x]$, the local density of interaction energy, is a periodic function of $x$ with the periodicity $P$. To quadratic order in $\stackrel{\leftrightarrow}{Q}, f_{S}[\stackrel{\leftrightarrow}{Q}, x]$ can be modeled as

$$
\begin{aligned}
f_{S}[\overleftrightarrow{Q}, x]= & g_{1}(x) \mathbf{z} \cdot \overleftrightarrow{Q} \cdot \mathbf{z}+g_{2}(x) \operatorname{Tr} \overleftrightarrow{Q}^{2}+g_{3}(x)(\mathbf{z} \cdot \overleftrightarrow{Q} \cdot \mathbf{z})^{2} \\
& +g_{4}(x) \mathbf{z} \cdot \overleftrightarrow{Q}^{2} \cdot \mathbf{z}
\end{aligned}
$$

so long as the local alignment axis of $f_{S}$ is normal to the substrate. In this work, we assume the textured pattern to consist of alternating stripes of equal width $P / 2$, with either the homeotropic or the planar aligning potential. Both alignment potentials are noted to have $z$ as the local ordering axis $[2,3]$. For the planar case, however, spontaneous symmetry breaking is a distinct possibility, which can lead to biaxial order in the boundary layer [3]. As shown in Refs. [2,3], the term linear in $\overleftrightarrow{Q}$ is dominant in either the homeotropic or the planar case. We thus adopt the following expression of $F_{S}$ :

$$
F_{S}=\int d^{3} \mathbf{r}\left[G(x) Q_{33} \delta(z)\right],
$$

where the surface coupling constant $G$ is noted to be a function of $x$. It is equal to $-G_{n}$ in the homeotropic region and to $G_{p}$ in the planar region, with $G_{n}, G_{p}>0$. 
Writing the total free energy $F=F_{B}+F_{S}$ in the dimensionless form as

$$
\Psi=\frac{F}{\Delta^{3} B^{4} / C^{3}}=\Psi_{B}+\Psi_{S},
$$

we obtain the bulk free energy

$$
\begin{aligned}
\Psi_{B}= & \int d^{3} \vec{\zeta}\left\{\left(t+\frac{1}{4}\right)\left(\frac{2}{3} \bar{Q}_{i j}^{2}\right)-\frac{4}{3} \bar{Q}_{i j} \bar{Q}_{j k} \bar{Q}_{k i}+\left(\frac{2}{3} \bar{Q}_{i j}^{2}\right)^{2}\right. \\
& \left.+\frac{2}{3+2 \rho} \bar{Q}_{i j, k}^{2}+\frac{2 \rho}{3+2 \rho} \bar{Q}_{i j, j} \bar{Q}_{i k, k}\right\},
\end{aligned}
$$

and the surface free energy

$$
\Psi_{S}=\int d^{3} \vec{\zeta}\left[g\left(\zeta_{1}\right) \bar{Q}_{33} \delta\left(\zeta_{3}\right)\right]
$$

Here $\bar{Q}_{i j}=C Q_{i j} / B$ is the dimensionless order parameter, $\Delta$ is the length unit that characterizes the distance over which the order parameter can vary significantly, $\vec{\zeta}=\mathbf{r} / \Delta$, $t=\left(T-T_{N I}\right) / 4\left(T_{N I}-T^{*}\right)$ with $4\left(T_{N I}-T^{*}\right)=B^{2} / a C$ being the temperature unit, $\rho=L_{2} / L_{1}$, and $g$ is the dimensionless surface coupling constant in the unit of $G_{0}=\Delta B^{3} / C^{2}$. For $5 \mathrm{CB}$ (where CB denotes cyano biphenyl), $a=0.065 \mathrm{~J} / \mathrm{cm}$ ${ }^{3} \mathrm{~K}, B=0.53 \mathrm{~J} / \mathrm{cm}^{3}, C=0.98 \mathrm{~J} / \mathrm{cm}^{3}, L=4.5 \times 10^{-14} \mathrm{~J} / \mathrm{cm}$, and $\rho \simeq 1 \quad[2,3]$. Accordingly, we have $\Delta=40 \AA$, $G_{0}=0.614 \mathrm{erg} / \mathrm{cm}^{2}$, and the temperature unit is $4.4 \mathrm{~K}$. The values of $g_{n}=0.05$ and $g_{p}=0.08$ are used in our numerical computation that follows [11].

\section{BULK ORIENTATIONAL STATES}

The LC equilibrium state is given by the order parameter configuration, which minimizes the free energy functional. To obtain the configuration $\bar{Q}_{i j}(\vec{\zeta})$, which minimizes the total free energy $\Psi$, we use the fourth order finite difference scheme to discretize the derivatives and employ the conjugate gradient method to seek the optimal configuration. Our experience shows that the global optimal state is invariably obtained starting from random configurations except near the first order transition point, to be specified below. Close to the transition point, the continuation method was applied to obtain the desired state, i.e., the optimal configuration at each step is used as the starting configuration for the next step. In this way, global optimal configuration in one regime may be used to obtain the metastable (locally optimal) configuration in other regime(s). Thermally stable and metastable states are thus identified around the transition point. Accuracy of the calculation is checked in two ways. First, the conjugate gradient method was found to minimize the free energy to an accuracy of $10^{-7}$. Second, by doubling the number of spatial points, we find the shift in minimized free energy is less than $0.2 \%$, while the shift in the critical values of the transition temperature or the critical texture periodicity are on the order of $2 \%$.

\section{A. Large- and small-periodicity limits}

Given a surface potential $f_{S}[\overleftrightarrow{Q}(z=0)]$, which is uniform in the $x y$ plane, the optimal $\bar{Q}_{i j}(\vec{\zeta})$ state is determined as follows. At fixed temperature $t<0$, minimizing $\left(t+\frac{1}{4}\right)\left(\frac{2}{3} \bar{Q}_{i j}^{2}\right)-\frac{4}{3} \bar{Q}_{i j} \bar{Q}_{j k} \bar{Q}_{k i}+\left(\frac{2}{3} \bar{Q}_{i j}^{2}\right)^{2}, \quad$ yields $\quad \bar{Q}_{i j}(z \rightarrow \infty)$ $=u(t)\left(3 n_{B i} n_{B j}-1\right) / 2$, with $u(t)=(3+\sqrt{1-32 t}) / 8$ and bulk director $\mathbf{n}_{B}$ to be determined by the condition that under the above boundary condition at $z=\infty$, the minimization of the total free energy functional should give the lowest free energy.

For the homeotropic aligning potential, the easy direction of alignment is normal to the surface while for the planar aligning potential, all in-plane directions are degenerate. Texturing the substrate into a stripe pattern thereby generates distinct domains of homeotropic or planar-aligned LC order close to the substrate, with domain walls separating the distinct alignment regions. In the $z$ direction, there are also two distinct regions of liquid crystalline order. Close to the substrate and extending about a few $P$ 's into the LC sample is the boundary layer region. Here the LC orientation tends to follow the local preferred directions of the substrate alignment potentials, with domain walls separating the homeotropic from the planar alignments. Extending from $z$ of a few $P$ 's to $z \rightarrow \infty$ is the bulk region. Here the domain walls disappear (since otherwise the elastic distortion energy would diverge), and the LC order is uniaxial, aligned along a bulk orientational director. The global optimal state for a textured stripe pattern substrate can be qualitatively deduced for the cases of $P \rightarrow 0$ and $P \rightarrow \infty$.

As $P \rightarrow \infty$, the directors in each potential region are able to adjust themselves in order to lower the surface energy. The cost is an appreciable elastic distortion in the domain walls separating the neighboring alignment regions. Numerically we obtain the optimal state with the bulk director lying in the $y z$ plane and making a nonzero tilt angle $\theta$ as measured from the $x y$ plane. The so-denoted $y z$ state's bulk director and the transition layer are illustrated schematically in Fig. 1. Why the $y z$ plane is selected as the easy direction in the planar regions is physically understandable because the twist elastic constant is always smaller than the splay and the bend elastic constants as long as $\rho>0$. Since the elastic distortion of the $y z$ state along the $x$ axis is mostly of twist character, the elastic distortion energy is thus minimized without having to increase the surface energy. Since the director far away from the substrate has to be uniform, it has to be in the $y z$ plane. This determines the $P \rightarrow \infty$ limiting state as the " $y z$ state."

As $P \rightarrow 0$, the textured substrate behaves as an effective medium since its periodicity is much shorter than the LC elastic correlation length. The director configuration close to the substrate is therefore nearly uniform and the bulk director is approximately given by the easy direction for a uniform substrate with surface interaction potential $\frac{1}{2}\left(g_{p}-g_{n}\right) \bar{Q}_{33}\left(\zeta_{3}=0\right)$. If $g_{p}>g_{n}$ as assumed in this work, the bulk director is expected to lie in the $x y$ plane. However, what should be the azimuthal angle $\phi$, measured from the $x$ axis, for the bulk director in the optimal state as $P \rightarrow 0$ ? From our numerical calculations, we find the global optimal state has the bulk director in the $x$ direction, denoted the $x$ 
state. The state with the bulk director in the $y$ direction, denoted the $y$ state, is metastable. Below we give a physical explanation for the stability of the $x$ state.

\section{B. Origin of the $x$ state: Rotational symmetry breaking and biaxiality}

To better understand the origin of the $x$ state, we first turn to the rotational symmetry properties of the system. Consider a uniform substrate with surface potential $f_{S}[\stackrel{\leftrightarrow}{Q}] \delta(z)$. In that case the spatial dependence of the order parameter is only along the $z$ direction, and the bulk free energy is invariant under the rotation about the $z$ axis, i.e., $F_{B}[\overleftrightarrow{Q}(\mathbf{r})]=F_{B}\left[R \overleftrightarrow{Q}(\mathbf{r}) R^{-1}\right]$, where $R$ is of the form

$$
\left[\begin{array}{ccc}
\cos \omega & \sin \omega & 0 \\
-\sin \omega & \cos \omega & 0 \\
0 & 0 & 1
\end{array}\right],
$$

and $R^{-1}$ is the inverse of $R$. Here $\omega$ denotes the rotation angle about the $z$ axis. If $f_{S}[\overleftrightarrow{Q}]$ is also rotationally invariant, as expressed by $f_{S}[\stackrel{\leftrightarrow}{Q}, x]$ in Eq. (4) without the $x$ dependence of the $g$ constants, then the whole system possesses rotational symmetry about the $z$ axis. Texturing the substrate introduces an $x$ dependence into $Q_{i j}$ and breaks the rotational symmetry of $F_{B}$ through the terms proportional to $L_{2}$ (i.e., if $L_{2}=0$, then $F_{B}$ would be rotationally invariant). The optimal $y z$ state in the large $P$ limit is a manifestation of the symmetry breaking due to the $L_{2}$ term since the three FrankOseen elastic constants obey $K_{\text {twist }}<K_{\text {splay }}=K_{\text {bend }}$ if $\rho>0$ (i.e., $L_{2}>0$ ).

The $L_{2}$ term is responsible for determining the bulk director in the optimal state as $P \rightarrow 0$. From Eq. (1), we find that if $Q_{12}(\mathbf{r})=Q_{23}(\mathbf{r})=0$, then functional differentiation yields

$$
\frac{\delta F}{\delta Q_{12}(\mathbf{r})}=0, \quad \frac{\delta F}{\delta Q_{23}(\mathbf{r})}=0,
$$

provided $Q_{i j, 2}=0$, which is always valid for the stripe pattern. That means it is possible to obtain a locally or globally optimal state with $Q_{12} \equiv 0$ and $Q_{23} \equiv 0$. The numerical results show that both the $x$ state and the $y$ state are indeed given by the three nonzero independent components $Q_{11}, Q_{22}$ and $Q_{13}$, with the bulk values $\bar{Q}_{11}=u, \bar{Q}_{22}=-u / 2, \bar{Q}_{13}=0$ in the $x$ state, and $\bar{Q}_{11}=-u / 2, \bar{Q}_{22}=u, \bar{Q}_{13}=0$ in the $y$ state. Close to the substrate, the order parameter deviates from the bulk value and exhibits spatial variation and biaxiality $\left(Q_{13}\right.$ $\neq 0)$ in response to the surface interaction potential, as schematically depicted in Fig. 1 for the $x$ state. To explain why the $x$ state can be lower in energy than the $y$ state, it is crucial to note that $\left|\bar{Q}_{13}\right|$ is always much larger in the $x$ state than in the $y$ state, as verified by numerical calculations. Physically this is reasonable because the order parameter along the $x$ direction, $Q_{11}$, is larger in the $x$ state. We turn to the explicit form of the $L_{2}$ term,

$$
\frac{1}{2} L_{2}\left[\left(Q_{11,1}+Q_{13,3}\right)^{2}+\left(Q_{31,1}+Q_{33,3}\right)^{2}\right],
$$

in which the two terms within the parentheses are always found to be opposite in sign, in either the $x$ state or the $y$ state. For biaxial distortion, the effective elastic constant in the $x$ state is therefore smaller than that in the $y$ state because of the larger $\left|Q_{13}\right|$, which in turn gives rise to a larger $\left|Q_{13}\right|$ for the $x$ state, as seen below. So the cause and consequence are consistent. Accordingly, the order parameters can better adjust to the the surface potential in the $x$ state, and the surface energy is therefore lowered as compared to the $y$ state.

A simple model can make concrete the above considerations. In either the $x$ or the $y$ state, the order parameter at $z=0$ deviates from its bulk value $\overleftrightarrow{Q}_{B}$. Suppose the total free energy of a uniform configuration $\overleftrightarrow{Q}(\mathbf{r})=\overleftrightarrow{Q}_{B}$, is denoted by $\mathcal{F}_{0}$. The total free energy in the $x$ or the $y$ state can be expressed as $\mathcal{F}=\mathcal{F}_{0}+\delta \mathcal{F}_{S}+\delta \mathcal{F}_{E}$, in which $\delta \mathcal{F}_{S}$ and $\delta \mathcal{F}_{E}$ are the surface and the elastic distortion energies caused by the deviation of $\stackrel{\leftrightarrow}{Q}(x, y, z=0)$ from $\stackrel{\leftrightarrow}{Q}_{B}$. In response to the surface interaction potential, such deviations always make $\delta \mathcal{F}_{S}<0, \delta \mathcal{F}_{E}>0$, and $\delta \mathcal{F}_{S}+\delta \mathcal{F}_{E}<0$, as seen below. We introduce a single parameter $q$, which measures the mean deviation of surface order parameters from the bulk value. $\delta \mathcal{F}_{S}$ and $\delta \mathcal{F}_{E}$ can thus be expressed as $c_{1} q$ and $c_{2} q^{2}$, respectively, with $c_{1}, c_{2}>0$. This is because $\delta \mathcal{F}_{S}$ can be positive or negative, depending on the direction of the deviation at surface, and is therefore directly associated with the sign of $q$. On the other hand, $\delta \mathcal{F}_{E}$ is always positive, since $F_{B}$ itself is minimized by $\stackrel{\leftrightarrow}{Q}(\mathbf{r})=\stackrel{\leftrightarrow}{Q}_{B}$. Therefore for small $|q|, \delta \mathcal{F}_{S}$ is linear in $q$ while $\delta \mathcal{F}_{E}$ is quadratic in $q$. By minimizing

$$
\mathcal{F}=\mathcal{F}_{0}+c_{1} q+c_{2} q^{2}
$$

we get $q_{s}=-c_{1} / 2 c_{2}$, with $\delta \mathcal{F}_{S}=-c_{1}^{2} / 2 c_{2} \quad$ and $\delta \mathcal{F}_{E}=c_{1}^{2} / 4 c_{2}$. It is readily seen that the smaller the effective elastic constant $c_{2}$, the better the surface adjustment of order parameters would be (which, among other things, would give rise to a larger $\left.\left|Q_{13}\right|\right)$. Consequently, the surface energy decreases while the elastic energy increases with increasing $c_{2}$. The total free energy $\delta \mathcal{F}=\delta \mathcal{F}_{0}-c_{1}^{2} / 4 c_{2}$, on the other hand, always decreases with decreasing $c_{2}$. These results are verified by our numerical solutions, which show that the surface energy is lower in the $x$ state, while the elastic energy is higher. The reverse is the case in the $y$ state. Just as in our simple explanation, the $x$ state is found to have the overall lower free energy. Thus the smaller effective elastic constant of the $x$ state, resulting from the biaxiality of the boundary layer, is the source of the equilibrium $x$ state in the $P \rightarrow 0$ limit.

\section{PHASE TRANSITIONS}

\section{A. The first order phase transition}

With the two $P \rightarrow \infty$ and $P \rightarrow 0$ limiting equilibrium states found to be distinct, a transition between the two is inevitable as $P$, or other relevant control parameter, is varied. Numerical calculations have been carried out only for $-2 \leqslant t<0$ because the Landau-de Gennes free energy expression becomes inaccurate far below the nematic-isotropic transition point. We find that in a wide range of $P$, the $y z$ state and the $x$ state can both exist, with one being stable (globally optimal) and the other metastable, while the $y$ state is always metastable. At a fixed temperature, as the texturing 


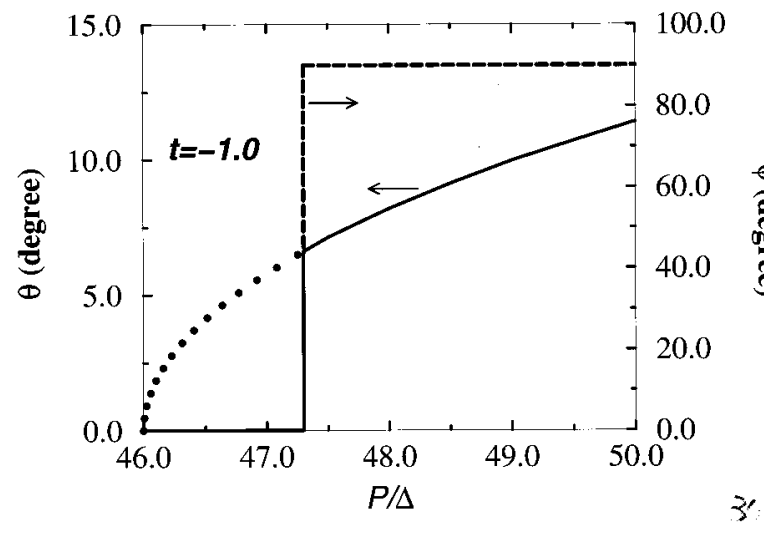

FIG. 2. Variation of the tilt angle $\theta$, measured from the $x y$ plane, and the azimuthal angle $\phi$, defined relative to the $x$ axis, plotted as a function of substrate texture periodicity $P$ at fixed $t=-1.0$. The solid line denotes the $\theta$ variation; the dashed line denotes the $\phi$ variation. The dotted line denotes the tilt angle variation of the metastable $y z$ state. A first order phase transition is noted at $P=47.3 \Delta$.

periodicity decreases, the bulk director of the $y z$ state gradually turns to the $x y$ plane and finally merges with the $y$ state when $P$ is sufficiently small. However, before this can happen, a first order transition from the $y z$ state to the $x$ state must occur since the $x$ state is the true ground state for $P \rightarrow 0$. In Fig. 2, the bulk director's tilt angle $\theta$ and azimuthal angle $\phi$ are depicted as a function of $P$, at fixed $t=-1.0$. It is noted that the first order transition occurs at $P_{c}=47.3 \Delta$. The same transition can be observed by varying the temperature around $t=-1.0$ if $P$ is fixed at some value close to $50 \Delta$. In Fig. 3, the tilt angle of the bulk director is depicted as a function of $t$, at fixed $P=47.5 \Delta$. The first order transition is noted to occur at $t=-1.017$. In Fig. 4 , the total free energies of the three orientational states are plotted as a function of $P$ at fixed $t=-1.0$. The existence of the first order phase transition at $P_{c}=47.3 \Delta$ is characterized by the crossing of the $y z$ state free energy with the $x$ state free energy, indicated by arrows.

The first order phase transition is physically understandable from the competition between surface and elastic ener-

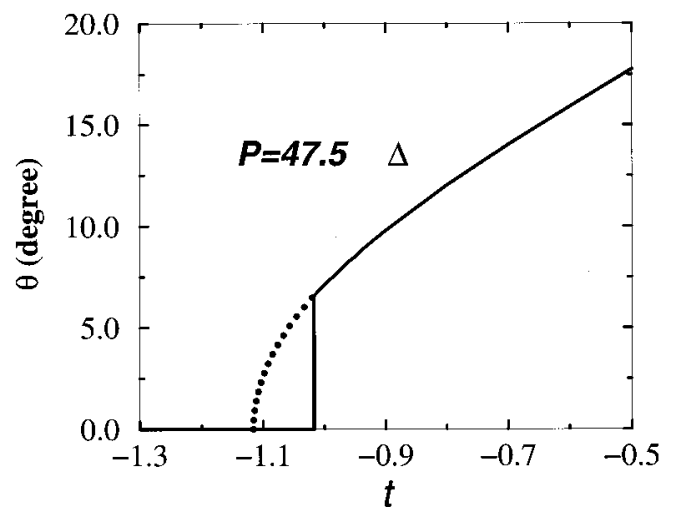

FIG. 3. Variation of the tilt angle $\theta$, plotted as a function of temperature $t$ for fixed $P=47.5 \Delta$. The dotted line denotes the tilt angle variation of the metastable $y z$ state. A first order phase transition is noted at $t=-1.017$.

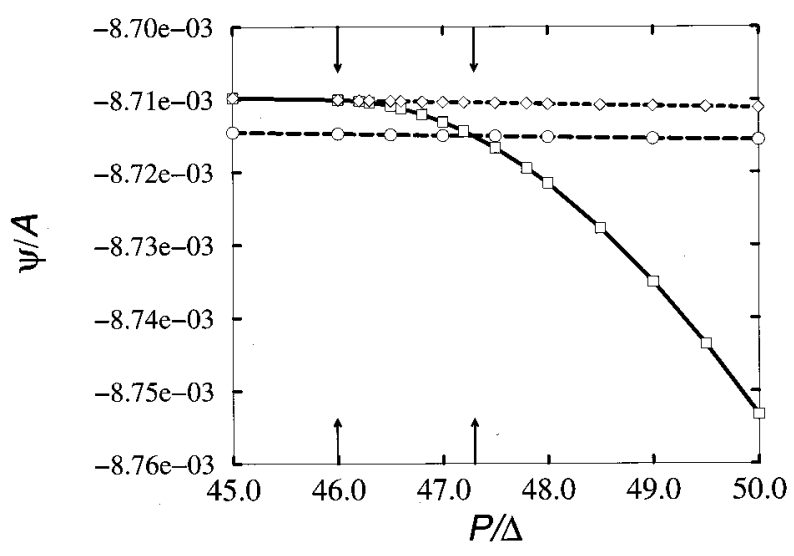

FIG. 4. Variation of the total free energy per unit area plotted as a function of substrate texture periodicity $P$ at fixed $t=-1.0 . \Psi$ is the dimensionless total free energy and $A$ is the dimensionless total area on which $\Psi$ is calculated. The solid line with open squares denotes the $y z$ state, the long-dashed line with open circles denotes the $x$ state, and the short-dashed line with open diamonds denotes the $y$ state. The arrows indicate the the first order phase transition at $P=47.3 \Delta$ and the virtual second order phase transition at $P=46.0 \Delta$.

gies. Compared with the $x$ state, the $y z$ state exhibits a larger elastic distortion but a better surface adjustment of the order parameters at the substrate. As $P$ decreases to the critical point $P_{c}$, the elastic distortion energy is increased to the extent that it becomes possible to achieve a lower overall energy by sacrificing the surface adjustment, if the elastic distortion energy can be lowered at the same time. This is in fact achieved by the $x$ state. From the point of view of lowering the temperature, a similar case can be made because lowering the temperature increases the uniaxial order parameter $u$. Since the three Frank-Oseen elastic constants are all proportional to $u^{2}$, the elastic distortion energy is thus increased. Meanwhile, the surface energy is proportional to $-u$. Consequently, as $t$ is lowered, one can have the same first order phase transition as decreasing $P$. A rough estimate shows that the elastic energy stored in the two adjacent stripes is proportional to $u^{2} P^{0}$. The surface energy, on the other hand, is roughly proportional to $-u P$. Hence the competition between the elastic energy and the surface energy leads to a critical periodicity $P_{c}$ approximately proportional to $u$. Therefore, lowering temperature increases $P_{c}$, and similarly for $T_{c}$. It is worthwhile to note that $P_{c}=47.3 \Delta$ at $t_{c}=-1.0 \quad$ and $\quad P_{c}^{\prime}=47.5 \Delta \quad$ at $t_{c}^{\prime}=-1.017$, so $P_{c}^{\prime} / P_{c} \cong\left[u\left(t_{c}^{\prime}\right) / u\left(t_{c}\right)\right]^{0.8}$. The above argument also leads to the prediction that the dimensionless critical periodicity $P_{c} / \Delta$ can be raised by choosing a smaller dimensionless surface coupling constant $g_{p}$, with $g_{p} / g_{n}$ and $\rho$ fixed, since the smaller $g_{p}$ is the less competitive the surface energy would be, and $P_{c} / \Delta$ is thereby raised by the relatively enhanced importance of the elastic distortion energy.

\section{B. The virtual second order phase transition}

On the small $P$ (or lower $T$ ) side of the first order phase transition, the $y z$ state is metastable. With further decrease in $P$ or $T$, the tilt angle in the $y z$ state approaches zero, and the 
$y z$ state merges with the $y$ state. This is shown by the tilt angle variation depicted in Figs. 2 and 3, and by the free energy variation plotted as a function of $P$ in Fig. 4. It can be regarded as a virtual second order phase transition because the order parameter configuration changes continuously, but a transition occurs in symmetry. From Figs. 2 and 4, it is noted that the virtual second order phase transition occurs at $P_{s}=46.0 \Delta$ for fixed $t=-1.0$. To elaborate on this point, we turn to the space-reversal symmetry of the system. With the origin of the coordinate system defined at the center of a stripe chosen arbitrarily, the free energy functional $F[\overleftrightarrow{Q}(\mathbf{r})]$ is invariant under the $x$-reversal transformation, i.e.,

$$
\begin{gathered}
Q_{11}(x, y, z) \rightarrow Q_{11}(-x, y, z), \quad Q_{22}(x, y, z) \rightarrow Q_{22}(-x, y, z), \\
Q_{12}(x, y, z) \rightarrow-Q_{12}(-x, y, z), \\
Q_{13}(x, y, z) \rightarrow-Q_{13}(-x, y, z), \\
Q_{23}(x, y, z) \rightarrow Q_{23}(-x, y, z),
\end{gathered}
$$

and also the $y$-reversal transformation, i.e.,

$$
\begin{aligned}
Q_{11}(x, y, z) \rightarrow & Q_{11}(x,-y, z), \quad Q_{22}(x, y, z) \rightarrow Q_{22}(x,-y, z), \\
& Q_{12}(x, y, z) \rightarrow-Q_{12}(x,-y, z), \\
& Q_{23}(x, y, z) \rightarrow-Q_{23}(x,-y, z), \\
& Q_{13}(x, y, z) \rightarrow Q_{13}(x,-y, z) .
\end{aligned}
$$

From numerical solutions, we find that if the bulk order parameter in any optimal state is invariant under either of the above two transformations, then the whole order parameter configuration is invariant under the same transformation. For the $y z$ state, since the bulk director is in the $y z$ plane, the bulk order parameter, with three nonzero independent components $Q_{11}, Q_{22}$, and $Q_{23}$, is invariant under the $x$-reversal transformation, and the whole configuration does show that $Q_{11}, Q_{22}$, and $Q_{23}$ are symmetric, while $Q_{12}$ and $Q_{13}$ are antisymmetric, with respect to $x$ reversal. For the $x$ and the $y$ states, the bulk order parameters, with the two nonzero independent components $Q_{11}$ and $Q_{22}$, are invariant under each of the two transformations, and the whole configuration shows that $Q_{12}$ and $Q_{23}$ vanish everywhere, while $Q_{11}$ and $Q_{22}$ are symmetric, $Q_{13}$ is antisymmetric, with respect to $x$ reversal. Here the $y$-reversal invariance and the uniformity in the $y$ direction lead to $Q_{12}=0$ and $Q_{23}=0$ while the $x$-reversal invariance results in the antisymmetric $Q_{13}$. As $\overleftrightarrow{Q}(z \rightarrow \infty)$ is uniform, any antisymmetric component with respect to $x$ reversal has to vanish at $z \rightarrow \infty$ in all the three states.

Due to the invariance of $F$ under the $y$-reversal transformation, there must be two degenerate $y z$ states, mutually related by Eq. (10). When the virtual second order phase transition occurs, the two $y z$ states become identical and merge into a single $y$ state. In the limit of small tilt angle $\theta>0$, the bulk order parameters of the two $y z$ states are given by $\bar{Q}_{11}^{B}=-u / 2, \quad \bar{Q}_{22}^{B}=u\left(1-3 \theta^{2} / 2\right)$, and $\bar{Q}_{23}^{B}$ $= \pm 3 \theta / 2$. The energy difference between the $y$ and the $y z$

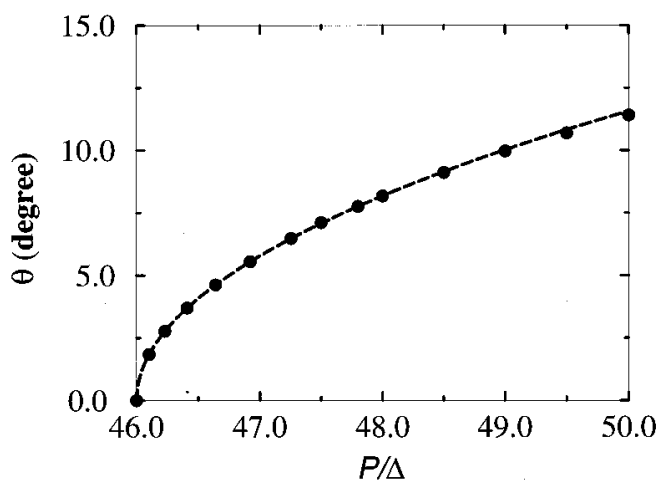

FIG. 5. Variation of the tilt angle $\theta$, plotted as a function of substrate texture periodicity $P$ in the proximity of the virtual second order phase transition point $P_{s}=46.0 \Delta$ at fixed $t=-1.0$. The solid circles represent the values of $\theta$ obtained through the minimization of total free energy. The dashed line represents a square root dependence on $P-P_{s}$.

states, $F_{y}-F_{y z}$, is thus an even function of bulk $Q_{23}^{B}$, and may be expanded in a power series of $Q_{23}^{B}$ as

$$
F_{y}-F_{y z}=R(P)\left(Q_{23}^{B}\right)^{2}+S(P)\left(Q_{23}^{B}\right)^{4},
$$

where $R(P)$ and $S(P)$ are $P$-dependent phenomenological parameters for a fixed temperature. The expansion is to the quartic order in $Q_{23}^{B}$, valid for small $\left|Q_{23}^{B}\right|$. From the numerical solutions, we find that close to the second order transition point $P_{s}, \theta$ is proportional to $\sqrt{P-P_{s}}$ while $F_{y}-F_{\mathrm{yz}}$ is proportional to $\left(P-P_{s}\right)^{2}$, as depicted in Figs. 5 and 6 . Such mean field behaviors can be obtained through a minimization of the free energy difference in Eq. (11), if we follow the Landau mean field approach for second order phase transition and choose $R(P)=r\left(P-P_{s}\right)$ and $S_{P}=S$, where $r$ and $S$ are $P$ independent. In case the periodicity is fixed, there are similar mean field behaviors for the tilt angle and the

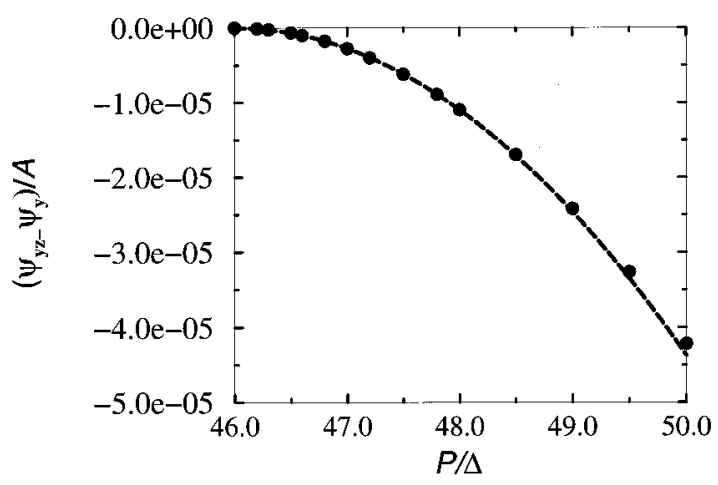

FIG. 6. Variation of the free energy difference, plotted as a function of substrate texture periodicity $P$ in the proximity of the virtual second order phase transition point $P_{s}=46 \Delta$ at fixed $t=-1.0$. The solid circles represent the free energy difference obtained in the minimization of total free energy. The dashed line delineates a quadratic dependence on $P-P_{s} . \Psi_{y z}$ and $\Psi_{y}$ are the dimensionless total free energies of the $y z$ and $y$ states, respectively, and $A$ denotes the dimensionless area of the substrate. 
energy difference, i.e., $\theta \propto \sqrt{T-T_{s}}$, and $F_{y}-F_{y z} \propto\left(T-T_{s}\right)^{2}$, which can be obtained by a similar Landau mean field assumption.

\section{The role of the tensor order parameter}

It is worthwhile to compare the present study with some prior works, which adopted the Frank-Oseen elastic free energy expression as the starting point. Barbero et al. [7] considered a solid surface textured into a periodic structure of wavelength $\lambda$ along the $x$ direction, with two alternating easy axes. They used a surface energy expression $f_{S}\left(\mathbf{n} \cdot \mathbf{n}_{e}\right)$ $=-\frac{1}{2} w\left(\mathbf{n} \cdot \mathbf{n}_{e}\right)^{2}$ in each of the two potential regions, where $\mathbf{n}_{e}$ is the easy axis, $\mathbf{n}$ is the director at the surface, and $w>0$ is the coupling constant. The bulk alignment thus obtained is only an effective easy direction. That is a direct consequence of the constrained variation of the order parameter, as pointed out in the Introduction. If we use a uniaxial order parameter $\overleftrightarrow{Q}=S(3 \mathbf{n n}-1) / 2$ with a fixed $S$ at a given temperature, then the Landau-de Gennes free energy expression is identical with the Frank-Oseen elastic distortion energy expression. Using the same uniaxial $\overleftrightarrow{Q}$, we also have the surface energies of the form $\frac{1}{2} w_{p}(\mathbf{n} \cdot \mathbf{z})^{2}$ and $-\frac{1}{2}$ $w_{n}(\mathbf{n} \cdot \mathbf{z})^{2}$ for the planar and the homeotropic potentials respectively, with $w_{p}, w_{n}>0$. A simplified formulation of the problem, of a similar form to that in Ref. [7], is thus derived. The only optimal state we can thus obtain is the $y z$ state, in which the tilt angle turns to the $x y$ plane gradually as $P$ or $t$ is lowered. With the $y z$ state as the only optimal state everywhere, no first order transition can be found. This shows that the tensor character of the liquid crystalline order is crucial in giving rise to the substrate-induced states and phase transitions discussed above.

\section{FIELD-INDUCED EFFECTS}

So far the orientational states and the phase transitions are discussed in the absence of any externally applied electric or magnetic field. In this section we consider the effects of an applied electric field. Effects due to magnetic field are expected to be similar and therefore would not be repeated. The electric field-induced free energy is [10]

$$
F_{E}=\int d \mathbf{r}\left\{-\frac{1}{8 \pi} \mathbf{E} \cdot \mathbf{D}\right\}
$$

where $\mathbf{D}$ is the electric displacement field, which is related to the electric field $\mathbf{E}$ by $\mathbf{D}=\overleftrightarrow{\epsilon} \mathbf{E} . \overleftrightarrow{\epsilon}$ is the local dielectric tensor, given by

$$
\stackrel{\leftrightarrow}{\epsilon}=\frac{1}{3}\left(\epsilon_{\|}+2 \epsilon_{\perp}\right)+\frac{2 \epsilon_{a}}{3} \overleftrightarrow{Q},
$$

where $\epsilon_{a}=\epsilon_{\|}-\epsilon_{\perp}$ is the maximum dielectric anisotropy. In the limit of small $\epsilon_{a}$, i.e., $\left|\epsilon_{a} / \epsilon_{\|}\right| \ll 1$, the electric field is almost uniform. We first discuss the effects of an electric field applied perpendicular to the substrate(s).

\section{A. The vertical field case}

For electric field applied perpendicular to the substrate, the field-induced free energy can be written in the dimensionless form as

$$
\Psi_{E}=-\frac{\epsilon_{a}}{\left|\epsilon_{a}\right|} \frac{E^{2}}{3 E_{0}^{2}} \int d \vec{\zeta} \bar{Q}_{33}(\vec{\zeta}),
$$

where $E_{0}=\sqrt{4 \pi B^{3} /\left|\epsilon_{a}\right| C^{2}}$ is the unit of field strength. Here we discuss the case of negative $\epsilon_{a}$. Since the $y z$ state has a finite tilt angle measured from the $x y$ plane, the vertical electric field is in favor of the $x$ state. For a sample of infinite thickness, the $x$ state is the only stable state as long as the field strength is nonzero. Here we consider the nematic liquid crystal in a cell of thickness $d$, sandwiched between two similarly textured substrates. In the regime $d \gg P$, the thickness of the boundary layer is much less than $d$, and the free energy contribution from the boundary layers is negligible. Thus we can use $\bar{Q}_{33}=u\left(3 n_{3} n_{3}-1\right) / 2$ with $u$ determined by $t$ only, and drop the $\mathbf{n}$-independent part in $\Psi_{E}$. In essence, the field-induced free energy is calculated within the FrankOseen framework. If the system is in the $x$ state, then $\Psi_{E}$ is almost zero since the bulk director is perpendicular to the $z$ direction. For the system in the $y z$ state, on the other hand, due to the finite tilt angle $\theta$ of the bulk $\mathbf{n}$, the field-induced free energy is positive (since $\epsilon_{a}<0$ ), and can be comparable to the difference between the free energies stored in the boundary layers of the $x$ and the $y z$ states. Suppose the energy difference between the $x$ and the $y z$ states, denoted by $\psi_{x}-\psi_{y z}=\delta \psi$, is positive for certain $P$ and $t$. The critical voltage $V_{c}$ is defined by the point at which $\Psi_{E} \simeq \delta \psi$. This mechanism of field-induced transition should be contrasted with that for the Fréedericksz transition, where the transition occurs when the elastic-field-induced correlation length, given by $\xi_{E} \sim \sqrt{4 \pi K_{\text {twist }}} /\left|\epsilon_{a}\right| S E^{2}$, is equal to or smaller than the cell thickness $d$. This implies that the critical voltage for the Freedericksz transition is independent of $d$. Here, if the critical voltage $V_{c}$ is lower than $V_{F}=\sqrt{4 \pi K_{\text {twist }} /\left|\epsilon_{a}\right| S}$, we observe that the field-induced distortion of the director configuration is very weak before the field-induced transition occurs, since for $V<V_{c}<V_{F}$ the field coherence length $\xi_{E} \sim \sqrt{4 \pi K_{\text {twist }}} /\left|\epsilon_{a}\right| S E^{2}$ would be larger than the cell thickness $d$. We can thus adopt first order perturbation approximation, i.e., the field-induced free energy in the $y z$ state is calculated by assuming the director configuration remains unchanged in the presence of the electric field to the zeroth order. The validity of such an approximation can be confirmed by the numerical results, which show that the change of the tilt angle at the middle plane is less than $7 \%$ when the applied voltage is close to but lower than $V_{F}$. So long as this approximation is valid, the field-induced energy $\Psi_{E}$ is proportional to $E^{2} d=V^{2} / d$, and the critical voltage $V_{c}$, obtained by setting $\Psi_{E}=\delta \psi$, is therefore proportional to $\sqrt{d}$. It follows that there exists a maximum cell thickness $d_{F}$ beyond which $V_{c}>V_{F}$, and the approximation would break down. If the starting $y z$ state is sufficiently close to the first order transition point, $d_{F}$ is found to be large and experimentally accessible. Below we make these considerations more quantitative. 


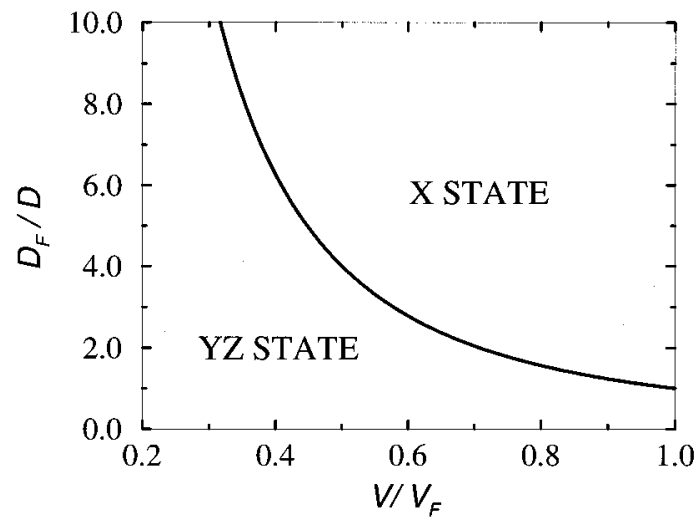

FIG. 7. Phase diagram of the two bulk orientational states plotted as a function of applied voltage $V$ and the inverse cell thickness $1 / D . V_{F}$ and $D_{F}$ are defined in the text.

The field-induced energy $\Psi_{E}$ is approximately given by

$$
\Psi_{E}=\frac{E^{2}}{2 E_{0}^{2}} u \sin ^{2} \theta A d / \Delta^{3},
$$

where $u=(3+\sqrt{1-32 t}) / 8, \theta$ is the tilt angle of the bulk director at middle of the cell, and $A$ is the cell area. By setting $\delta \psi$ equal to $\Psi_{E}$ at $V=V_{c}$, we obtain

$$
V_{c}=\sqrt{\frac{2}{u \sin ^{2} \theta}\left(\frac{\delta \psi}{A / \Delta^{2}}\right) \frac{d}{\Delta}} E_{0} \Delta .
$$

To determine $d_{F}$, we note that $V_{F}$ can be expressed as

$$
V_{F}=E_{0} \Delta \sqrt{\frac{18 L_{1}}{3 L_{1}+2 L_{2}} u},
$$

where $K_{\text {twist }}$ is written as $9 L_{1} S^{2} / 2$. Equating $V_{c}$ and $V_{F}$ yields

$$
d_{F}=\Delta u^{2} \frac{9}{3+2 \rho} \frac{\sin ^{2} \theta}{\delta \psi /\left(A / \Delta^{2}\right)} .
$$

For $P=50.0 \Delta$ and $t=-1.0$, we have $\theta=11.42^{\circ}$ and $\delta \psi=3.772 \times 10^{-5} A / \Delta^{2}$, from which one obtains $d_{F}=2.2 \times 10^{3} \Delta$. For most nematic liquid crystals, $\Delta$ ranges from several tens of to a few hundred angstroms, therefore $d_{F}$ can be larger than $10 \mu \mathrm{m}$. With $\left|\epsilon_{a}\right| \sim 0.1, V_{F}$ is usually several volts, as is well known for the Fréedericksz transition $[10,12]$. Thus the field-induced transition can be easily realized, provided the textured substrates can be properly prepared. In Fig. 7, the dependence of $V_{c}$ on $d$ is depicted for $d_{F} / 10 \leqslant d \leqslant d_{F}$. Here $V_{c}$ and the field-induced transition are calculated by minimizing $\Psi_{B}+\Psi_{S}+\Psi_{E}$. It is seen that the heuristic relation $V_{c} \propto \sqrt{d}$, derived above, is well followed. The $x$ state in the upper right region is separated from the $y z$ state in the lower left region by a first order field-induced transition.

For $V<V_{F}$, the field correlation length $\xi_{E}$ has been noted to be larger than the cell thickness. Consequently, the director configuration of the $y z$ state remains almost invariant when the field is turned on. However, due to the existence of the $x$ state, which is a little higher in energy than the $y z$ state at zero voltage, an abrupt change of the whole configuration can occur at a moderate voltage $V \leqslant V_{F}$. In this case the transition is actually determined by the boundary layer and not by the field coherence length. This is not surprising since the surface anchoring is effectively weak due to the proximity to the phase transition point. When the starting $y z$ state is located far away from the transition point, the $x$ state becomes inaccessible and the surface anchoring becomes effectively strong. We conclude that microtextured substrates can provide the effective tuning of anchoring strength, varying continuously from the strong (away from the transition point) to the weak (close to the transition point).

\section{B. The transverse field case}

For electric field applied parallel to the substrate, the field-induced free energy can also be calculated within the Frank-Oseen framework. It is clear that the orientational transitions can be induced by an in-plane field as well, depending on its specific direction and the sign of $\epsilon_{a}$. If $\epsilon_{a}>0$, a transition from the $y z$ state to the $x$ state can be induced by a field in the $x$ direction. If $\epsilon_{a}<0$, the same field can induce a transition from the $x$ state to the $y z$ state. The same is true for a field in the $y$ direction. In particular, if $P \rightarrow 0$, then the bulk directors of the two states are both in plane. Consequently, the vertical field can no longer induce any transition, while the transverse field is still effective.

In the $P \rightarrow 0$ limit, the energy difference between the $x$ and the $y$ states is quite small. For $t=-1.0$ and $P=45.0 \Delta$, we have $\psi_{y}-\psi_{x} \simeq 4.6 \times 10^{-6} A / \Delta^{2}$, which is much smaller than $\psi_{x}-\psi_{y z} \simeq 3.8 \times 10^{-5} \mathrm{~A} / \Delta^{2}$ for $t=-1.0$ and $P$ $=50.0 \Delta$, used in the previous subsection. For a uniform transverse field in the $y$ direction, a transition from the $x$ state to the $y$ state can thus occur at the critical field strength

$$
E_{c}=E_{0} \sqrt{\frac{2}{u} \frac{\psi_{y}-\psi_{x}}{A / \Delta^{2}} \frac{1}{d / \Delta}}
$$

Using $\epsilon_{a} \sim 0.1$ and $d \sim 10 \mu \mathrm{m}$, we find $E_{c} d \simeq 0.24 \mathrm{~V} \ll V_{F}$. This implies that the anchoring by the textured substrate is effectively very weak in the small periodicity limit.

\section{CONCLUDING REMARKS}

What we have presented in this paper only serves to illustrate the possible orientational states and phase transitions associated with a class of substrates. It is obvious that the stripe pattern of alternating planar and homeotropic aligning conditions by no means exhausts all possible patterns of microtextures. For example, a stripe pattern of alternating homogeneous aligning conditions of equal width, with neighboring easy directions orthogonal to each other, can induce two degenerate orientational states with bulk directors parallel to the substrate but orthogonal to each other. In particular, the effective anchoring strength can also be tuned by varying the texturing periodicity and/or the temperature. In addition, two-dimensional textures are also of interest.

The dynamics associated with the field-induced orientational transitions is yet to be investigated. Since the anchor- 
ing by textured substrates can be effectively weak, it is of special interest to investigate the transition dynamics in the boundary layer induced by voltages lower than that of the Fréedericksz transition. Work in this direction is presently under way.

\section{ACKNOWLEDGMENTS}

One of us (T.Z.Q.) was supported by the Hong Kong ITDC Grant No. AF/155/94. We wish to thank H. S. Kwok, M. Loy, and Y. Liao for helpful discussion.
[1] H. Hsiung, Th. Rasing, and Y. R. Shen, Phys. Rev. Lett. 57, 3065 (1986); W. Chen, L. J. Martinez-Miranda, H. Hsiung, and Y. R. Shen, ibid. 62, 1860 (1989).

[2] P. Sheng, Phys. Rev. A 26, 1610 (1982).

[3] T. J. Sluckin and A. Poniewierski, Phys. Rev. Lett. 55, 2907 (1985); A. K. Sen and D. E. Sullivan, Phys. Rev. A 35, 1391 (1987); P. Sheng, B. Z. Li, M. Y. Zhou, T. Moses, and Y. R. Shen, ibid. 46, 946 (1992); Y. L'vov, R. M. Hornreich, and D. W. Allender, Phys. Rev. E 48, 1115 (1993).

[4] X. Zhuang. L. Marrucci, and Y. R. Shen, Phys. Rev. Lett. 73, 1513 (1994).

[5] D. W. Berreman, Phys. Rev. Lett. 28, 1683 (1972).

[6] S. Faetti, Phys. Rev. A 36, 408 (1987).

[7] G. Barbero, T. Beica, A. L. Alexe-Ionescu, and R. Moldovan, J. Phys. II (France) 2, 2011 (1992).

[8] In a field theory with the free energy density of the form $k(\nabla \phi)^{2}+m \phi^{2}$, the elastic correlation length is $\sqrt{k / m}$. The
Frank-Oseen theory only considers the gradient terms and the elastic correlation length is thus infinite.

[9] T. Z. Qian and P. Sheng, Phys. Rev. Lett. 77, 4564 (1996).

[10] P. Sheng and E. B. Priestley, in Introduction to Liquid Crystals, edited by E. B. Priestley, P. J. Wojtowicz, and P. Sheng (Plenum Press, New York, 1975), p. 153.

[11] In Ref. [1], the value of $g_{n}$ obtained is $<0.08$ for 5CB. Our choice is in agreement with their experimental results. The value of $g_{p}$ is not known accurately. However, its effect on the induced orientational states can always be maintained by making the width of the planar stripes proportionally larger (smaller) than the homeotropic stripes if the true value of $g_{p}$ is smaller (larger) than the one chosen.

[12] This is a typical value for the maximum dielectric anisotropy. See, for example, P. G. de Gennes and J. Prost, The Physics of Liquid Crystals (Oxford University Press, New York, 1993). 